\title{
Planning and Dosimetric Study of Volumetric Modulated Arc Based Hypofractionated Stereotactic Radiotherapy for Acoustic Schwannoma - 6MV Flattening Filter Free Photon Beam
}

\author{
Shanmugam Thirumalai Swamy ${ }^{1,2 *}$, Chandrasekaran Anu Radha ${ }^{2}$, Gandhi \\ Arun $^{1}$, Murugesan Kathirvel ${ }^{1}$, Sai Subramanian' ${ }^{1}$
}

\begin{abstract}
Background: The purpose of this study was to assess the dosimetric and clinical feasibility of volumetric modulated arc based hypofractionated stereotactic radiotherapy (RapidArc) treatment for large acoustic schwannoma (AS >10cc). Materials and Methods: Ten AS patients were immobilized using BrainLab mask. They were subject to multimodality imaging (magnetic resonance and computed tomography) to contour target and organs at risk (brainstem and cochlea). Volumetric modulated arc therapy (VMAT) based stereotactic plans were optimized in Eclipse (V11) treatment planning system (TPS) using progressive resolution optimizer-III and final dose calculations were performed using analytical anisotropic algorithm with $1.5 \mathrm{~mm}$ grid resolution. All AS presented in this study were treated with VMAT based HSRT to a total dose of 25Gy in 5 fractions (5fractions/ week). VMAT plan contains 2-4 non-coplanar arcs. Treatment planning was performed to achieve at least $99 \%$ of PTV volume (D99) receives $100 \%$ of prescription dose $(25 \mathrm{~Gy})$, while dose to OAR's were kept below the tolerance limits. Dose-volume histograms (DVH) were analyzed to assess plan quality. Treatments were delivered using upgraded $6 \mathrm{MV}$ un-flattened photon beam (FFF) from Clinac-iX machine. Extensive pretreatment quality assurance measurements were carried out to report on quality of delivery. Point dosimetry was performed using three different detectors, which includes CC13 ion-chamber, Exradin A14 ion-chamber and Exradin W1 plastic scintillator detector (PSD) which have measuring volume of $0.13 \mathrm{~cm}^{3}, 0.009 \mathrm{~cm}^{3}$ and $0.002 \mathrm{~cm}^{3}$ respectively. Results: Average PTV volume of AS was $11.3 \mathrm{cc}( \pm 4.8)$, and located in eloquent areas. VMAT plans provided complete PTV coverage with average conformity index of $1.06( \pm 0.05)$. OAR's dose were kept below tolerance limit recommend by American Association of Physicist in Medicine task group-101(brainstem $V_{0.5 c c}<23 G y$, cochlea maximum < 25Gy and Optic pathway <25Gy). PSD resulted in superior dosimetric accuracy compared with other two detectors ( $p=0.021$ for PSD
\end{abstract}

Keywords: Acoustic schwannoma - VMAT - HSRT - point dosimetry - COMPASS

Asian Pac J Cancer Prev, 16 (12), 5019-5024

\section{Introduction}

Stereotactic radiosurgery (SRS) and stereotactic radiotherapy (SRT) have been used for several decades in the treatment of benign and malignant lesions as well as functional disorders. The major feature that separates stereotactic treatment from conventional radiation treatment is the delivery of large doses in one or few fractions, which results in a high biological effective dose (BED) (Ingrosso et al., 2012). In order to minimize the normal tissue toxicity, conformation of high doses to the target and rapid fall-off doses away from the target is critical. Current hypofractionated stereotactic radiotherapy (HSRT) protocols generally involve 3-5 treatments with a dose of 5-22 Gy per fraction to sites such as the brain, spine, liver, and lung (Benedict et al., 2010). The desired biological effect is achieved both by fractionation and perhaps more importantly by the differential dose delivered to targeted and normal tissue; the goal is to minimize the volume of normal tissue exposed to a high dose of radiation (Subramanian et al., 2012). Acoustic schwannoma (AS) (also known as vestibular schwannoma) is a benign tumor that originates from the vestibular portion of the eighth cranial nerve. Management options were observation, microsurgery, SRS and HSRT. The SRS (12-14Gy in single fraction) approach to the treatment of small to medium sized AS was well consolidated in literature with results demonstrating both very high local control rates (larger than $90 \%$ up to $96-98 \%$ ) as well as minimal toxicity (5 year radiation related toxicity as low as $\sim 5-10 \%$ ) (Kappor et al., 2011). But for large AS and in eloquent areas (brainstem and cochlea) HSRT will be better choice. HSRT will have good control rates and low side effects than SRS (Wang et al., 2012). This technique 
combines the physical advantage of stereotaxy and the radiobiologic advantage of fractionation. In this study we have evaluated volumetric modulated arc therapy (VMAT) for the treatment of these lesions (AS) with HSRT.

Initially stereotactic treatment was delivered using $\mathrm{X}$-knife (cones and micro multi leaf collimators) and Gamma knife, which consumes more treatment time (Abacioglu et al., 2014). In 2008, Volumetric Modulated Arc Therapy (VMAT) was introduced into clinical practice, which produces superior/comparable dose distribution than conventional fixed field intensity modulated radiotherapy (IMRT) (Ekambram et al., 2015). Many studies have proven that VMAT (RapidArc) significantly reduce treatment time compared with conventional IMRT (Swamy et al., 2014) and other advanced methods (Tomotherapy/ Cyber knife). VMAT (RapidArc) based stereotactic treatment produces highly conformal dose distribution by simultaneously changing MLC position, dose rate and gantry speed during patient treatment. Wolff et al., (2010) have shown that RapidArc based SRS for vestibular schwannoma would improve the planning target volume (PTV) conformity and reduce the treatment time compared to conventional cone based SRS. The requirements of large doses and highly accurate targeting in HSRT mean that special attention needs to be paid to all aspects of the treatment for each patient, including immobilization, localization, pre-treatment dose verification and review of on-board imaging by the physician (Benedict et al., 2010). Many studies have shown that SRS will have good control rates and low side effects for small and medium sized AS (Kappor et al., 2011). The aim of the present study was to report the clinical feasibility as well as the dosimetric benefit of volumetric modulated arc based hypofractionated stereotactic radiotherapy for large acoustic schwannoma and in eloquent areas. As recommended by American Association of Physicist in Medicine (AAPM) report-85, we have performed pretreatment quality assurance of VMAT plan by using three different detectors and secondary TPS verification using COMPASS 3D dosimetry system.

Pretreatment QA was performed in a phantom using chamber, film, 2D array and electronic portal imaging. Each of these devices has been proven useful but has its own limitations (Xin et al., 2012; Thirumalai et al., 2014). Due to involvement of small field size the point dosimetry would be better choice than 2D planar dosimetry. For point dose verification, ion chambers are considered to be the ideal choice because of their excellent stability, linear response to absorbed dose, small directional dependence, beam-quality response independence, and traceability to a primary calibration standard. However, for stereotactic treatments, high-spatial resolution is important for accurate dose measurement (Gagnon et al., 2012). The deviation between measured and actual dose values in small fields are created by a combination of several phenomena: the effects of volume dose averaging resulting from the finite size of the detector, the subsequent perturbation caused by the detector itself, and the disruption of charged particle equilibrium caused by small effective source sizes (Gagnon et al., 2012). Due to these multiple complications, radiosurgery field dosimetry remains a challenging task, and there is still no definite detector that can be considered ideal for SRS quality assurance (QA). In addition to dosimetric analyses, we have compared three different detectors for verification of point dose in patient-specific quality assurance of VMAT based stereotactic treatments.

\section{Materials and Methods}

\section{Image acquisition and contouring}

Ten cases of acoustic schwannoma (9 left side and1 right side) were selected for this study, where surgery and SRS were not possible due to target size and close location to cochlea and/or brainstem. These patients were immobilized using BrainLab (Type-B) mask and computed tomography (CT) scans was performed with localizer. CT scans with contrast were acquired with 1 $\mathrm{mm}$ slice thickness covering the entire head of the patients in Biograph 16 Slice PET-CT scanner (Siemens Medical Systems Concord, CA). A planning magnetic resonance (MR) scan was performed with a three-dimensional (3D) fast spoiled gradient recovery sequence in which axial post contrast MR sections were obtained with $1.0 \mathrm{~mm}$ slice thickness and no inter slice gap. Two imaging sequences were transferred to the Eclipse (V11) Treatment Planning System (TPS) (Varian Medical Systems, Palo Alto, CA, USA) and were fused for delineation of target and organs at risk. A margin of 1-2 mm was added to generate the PTV. For these ten patients PTV volume was ranging from $3 \mathrm{cc}$ to $18.0 \mathrm{cc}$. OAR's such as brainstem, cochlea, lens, retina, optic nerves and optic chiasm were delineated. Contoured images were then localized for SRT coordinates in the iPlan (V4.1.1) TPS (BrainLab, Feldkirchen, Germany) after Digital Imaging and Communications in Medicine (DICOM) based data transfer between the systems. The iso-center was positioned in the center of the target volume and data sent back to Eclipse TPS for planning.

\section{VMAT Planning and Delivery}

VMAT based stereotactic plans were optimized in Eclipse TPS using progressive resolution optimizerIII and final dose calculations were performed using analytical anisotropic algorithm (AAA) with $1.5 \mathrm{~mm}$ grid resolution. All AS presented in this study were treated with VMAT based HSRT to a total dose of 25Gy in 5 fractions (5fractions/week). VMAT plans contain 2 to 4 non-coplanar arcs. Treatment planning was performed to achieve at least $99 \%$ of PTV volume (D99) receives $100 \%$ of prescription dose (25Gy), while dose to OAR's were kept within tolerance limits. Dose-volume histogram (DVH) of VMAT plans were analyzed for these ten patients. In particular, for PTV Conformity index (C.I) and OAR's maximum dose. The C.I is defined as the ratio between prescribed dose volume and PTV volume. For organs at risk, brainstem dose to $0.5 \mathrm{cc}$ volume $\left(\mathrm{V}_{0.5 \mathrm{cc}}\right)$ were also analyzed.

Treatments were delivered using upgrades $6 \mathrm{MV}$ unflattened photon beam from Clinac-iX (Varian Medical Systems, Palo Alto, USA). The machine was equipped with millennium 120 multi-leaf collimator (MLC), on- 
board imager (OBI) and maximum dose rate of $1400 \mathrm{MU} /$ min. Beam parameters (gun current, voltage, etc.,) remain same for flattened and un-flattened beam, by removing the flattening filter from beam path has increase the dose rate from 600 to $1400 \mathrm{MU} / \mathrm{min}$. Target positioning printouts were generated from iPlan TPS and patients were aligned precisely on the treatment couch using the BrainLab positioning box and 6D mount. Treatment was executed after image verification with 2D-2D matching of kilo voltage-kilo voltage $(\mathrm{kV}-\mathrm{kV})$ planar images and 3D matching of cone beam computed tomography (CBCT) acquired with the Varian OBI system installed at linear accelerator. For non coplanar arcs, 2D MV image from Varian amorphous silicon (aSi1000) portal cassette was used to verify the patient position.

\section{Pre-treatment $Q A$}

Pre-treatment QA was performed in two different context (i) point dosimetry using three different detectors (ii) independent secondary TPS dose verification using COMPASS 3D dosimetry system. In point dosimetry, to compare measurements and TPS dose calculations, the three different set of phantom with the detector inserted was CT scanned and the verification plans were then created on this CT dataset. This includes CC13 ionchamber (Iba, Schwarzenbruck, Germany), Exradin A14 ion-chamber (Standard Imaging, Middleton, USA) and Exradin W1 plastic scintillator detector (PSD) (Standard Imaging, Middleton, USA) which have measuring volume of $0.13 \mathrm{~cm}^{3}, 0.009 \mathrm{~cm}^{3}$ and $0.002 \mathrm{~cm}^{3}$ respectively. Near water equivalence and high spatial resolution of PSD makes better choice for small field's dose measurements than ion-chamber (Figure 1). Verification plans were created in Eclipse TPS for clinically approved plan. Dose calculation was performed using AAA algorithm with grid size of $1.5 \mathrm{~mm}$ and couch was reset to zero. The absolute dose measured at iso-center was compared with the TPS calculated dose. Percentage of variation between measured and calculated was using formula [(measured dose-TPS dose)/TPS dose $\times 100]$. Statistical analyses were performed using the Student's t-test and differences were considered to be significant for $\mathrm{p}$-value $<0.05$. Placing small volume chamber in iso-centre play crucial role, so before measurement, CBCT was used to localize the chamber accurately with respect to the linear accelerator. Crosscalibration of detectors and electrometer was performed prior to measurements using a calibrated radiation beam with corresponding field size. For independent secondary TPS dose verification, ten VMAT-HSRT plans were

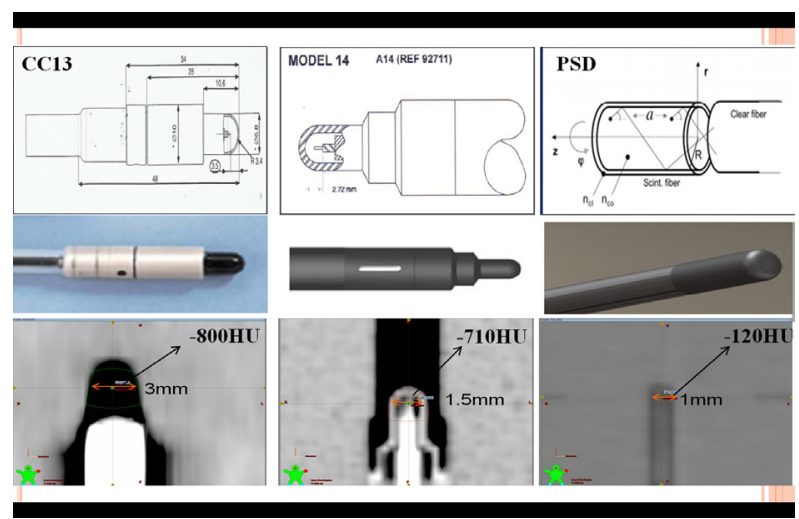

Figure 1. Near Water Equivalence and High Spatial Resolution of PSD Makes Better Choice for Small Field's Dose Measurements rather than Ion-chamber CC13 and A14



Figure 2. Isodose Distributions in Three Planes and Dose Volume Histogram view for One Sample Case of Acoustic Schwannoma having Planning Target Volume of $13.8 \mathrm{cc}$ 


\section{Shanmugam Thirumalai Swamy et al}

transferred to the COMPASS (V3) 3D dose verification system, in which the re-computation of the dose were performed using collapsed cone convolution/superposition (CCC/S) algorithm. COMPASS allows to compare the 3D dose distribution and DVH between COMPASS computed and TPS (Eclipse) calculated. The average doses for PTV and organs at risk (OAR's) in ten patients were compared between TPS calculated and COMPASS computed. The average 3D global gamma was calculated using criteria of $3 \mathrm{~mm}$ distance to agreement (DTA) and 3\% dose difference (DD). OAR1 and OAR2 was concentric ring like structures of $5 \mathrm{~mm}$ and $10 \mathrm{~mm}$ thickness and cropped at a distance of $5 \mathrm{~mm}$ and $10 \mathrm{~mm}$ from PTV respectively.

\section{Results}

In our study of acoustic schwannoma, tumors were large sized and/or abutting brainstem. Figure 2 shows example of dose distributions for one acoustic schwannoma patient with a PTV volume of $13.8 \mathrm{cc}$ in axial, coronal, and sagittal planes. In table 1 , for all ten patients PTV C.I was less than radiation therapy oncology group (RTOG-915) recommended value of 1.2, for two patients the values were less than 1 , due to large portion of tumor abutting the brainstem. Two patients left cochlea dose were found to be more than tolerance dose of $25 \mathrm{~Gy}$, as tumor was extending into cochlea and patient already had hearing loss. For brainstem, maximum and $\mathrm{V}_{0.5 \mathrm{cc}}$ dose was less than tolerance dose of $31 \mathrm{~Gy}$ and $23 \mathrm{~Gy}$ recommended by AAPM task group (TG)-101 (Benedict et al., 2010) respectively. Optic pathway structure encompasses the right optic nerve, left optic nerve and optic chiasm. For all ten patients optic pathway was located far from tumor, so dose to these structures was well within tolerance limit of $25 \mathrm{~Gy}$. The maximum dose of contralateral cochlea, left retina, right retina, left lens and right lens were in range of 307-572cGy, 193-604cGy, 125-376cGy, 127-402cGy and 38-204cGy respectively. Treatments were successfully performed for all patients without specific technical or clinical issues. With a median follow-up of 6 months, none of these patients developed acute toxicity. Delayed toxicity and treatment outcome such as obliteration rates cannot be reported at this early stage and require a follow-up of at least 18-36 months for complete assessment (Subramanian et al., 2012).

The percentage difference between TPS calculated and measured for three different detectors were shown in table 2. The mean percentage difference of PSD was less than A14 and CC13. For all ten patients, PSD shows less deviations compared to other two ion-chambers. PSD measured point doses were within $\pm 3 \%$ of TPS calculated. The PSD resulted in superior dosimetric accuracy compared with other two detectors ( $\mathrm{p}=0.021$ for PSD $v s$ $\mathrm{A} 14, \mathrm{p}<0.005$ for PSD vs CC13). There was no statistical difference in results between A14 and CC13 ( $\mathrm{p}=0.0685$ ). In VMAT based stereotactic treatment because of small beamlets involvement it is recommended to validate TPS calculated dose using independent secondary 3D dose verification system. The COMPASS uses plan from Eclipse TPS and inbuilt beam modeling to calculate the dose in the imported patient CT data. Figure 3 shows isodose distribution and DVH comparison between Eclipse TPS calculated and COMPASS computed. In table 3, 10 patients percentage difference of average dose and average 3D gamma between Eclipse TPS calculated and COMPASS computed for PTV and critical OAR's were listed. Maximum percentage deviation of PTV, OAR1 and OAR2 were $2.79 \%, 4.51 \%$ and $4.73 \%$ respectively. For 10 plans, the average 3D gamma between TPS calculated and COMPASS computed for PTV, OAR1 and OAR2

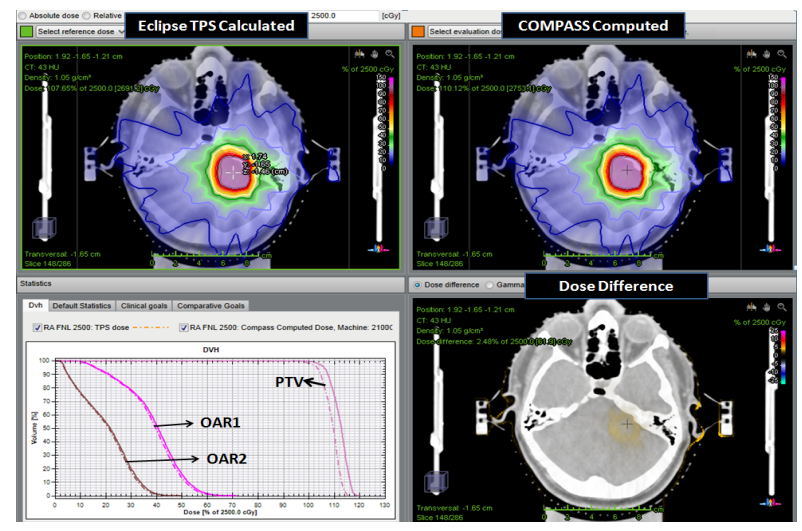

Figure 3. Dose Distribution and DVH Comparison between Eclipse TPS Calculated and COMPASS Computed for an Acoustic Schwannoma Patient

Table 2. Dose Difference between Eclipse TPS Calculated and Measured using Three Different Detectors

\begin{tabular}{|c|c|c|c|c|c|c|c|c|c|}
\hline \multirow[t]{2}{*}{ Pt Id } & \multicolumn{3}{|c|}{ CC13 (volume $0.13 \mathrm{cc}$ ) } & \multicolumn{3}{|c|}{ A14 (volume $0.009 \mathrm{cc}$ ) } & \multicolumn{3}{|c|}{ PSD (volume $0.002 \mathrm{cc}$ ) } \\
\hline & $\begin{array}{c}\text { TPS } \\
\text { dose cGy }\end{array}$ & $\begin{array}{l}\text { Measured } \\
\text { dose cGy }\end{array}$ & $\% \mathrm{Var}$ & $\begin{array}{c}\text { TPS } \\
\text { dose cGy }\end{array}$ & $\begin{array}{l}\text { Measured } \\
\text { dose cGy }\end{array}$ & $\% \operatorname{Var}$ & $\begin{array}{c}\text { TPS } \\
\text { dose cGy }\end{array}$ & $\begin{array}{l}\text { Measured } \\
\text { dose cGy }\end{array}$ & $\% \operatorname{Var}$ \\
\hline 1 & 571.1 & 545 & -4.6 & 585.6 & 570.06 & -2.7 & 585.2 & 578.9 & -1.1 \\
\hline 2 & 628.5 & 603.6 & -4 & 643.4 & 610.7 & -5.1 & 651.3 & 650.1 & -0.2 \\
\hline 3 & 548.6 & 524.8 & -4.3 & 563.6 & 546.4 & -3.1 & 564.9 & 562.95 & -0.3 \\
\hline 4 & 553.6 & 537.7 & -2.9 & 572.8 & 571.5 & -0.2 & 558 & 565.61 & 1.4 \\
\hline 5 & 503.7 & 492.5 & -2.2 & 523.9 & 507.4 & -3.1 & 508 & 505.96 & -0.4 \\
\hline 6 & 554.6 & 535.9 & -3.4 & 567.2 & 551.1 & -2.8 & 554.8 & 540.35 & -2.6 \\
\hline 7 & 589.6 & 579.1 & -1.8 & 611.2 & 596.1 & -2.5 & 598.1 & 594.6 & -0.6 \\
\hline 8 & 605.3 & 595.7 & -1.6 & 620.7 & 639.5 & 3 & 610 & 620.34 & 1.7 \\
\hline 9 & 558.3 & 544.9 & -2.4 & 576.1 & 582.2 & 1.1 & 563.8 & 580.15 & 2.9 \\
\hline 10 & 472.1 & 466.5 & -1.2 & 491.3 & 489.5 & -0.4 & 475.4 & 470.9 & -0.9 \\
\hline Avg & & & -2.5 & & & -1.6 & & & -0.01 \\
\hline SD & & & 1.19 & & & 2.41 & & & 1.6 \\
\hline
\end{tabular}

*Avg- Average, SD- Standard deviation, Var- Variation 
Table 3. Average 3D Gamma $(\lambda)$ and Mean Dose Volume Difference between Eclipse TPS Calculated (TPS) and COMPASS Computed (COM) for 10 AS Patients PTV and OAR'S

\begin{tabular}{|c|c|c|c|c|c|c|c|c|c|c|c|c|}
\hline \multirow{3}{*}{$\begin{array}{l}\mathrm{Pt} \\
\mathrm{Id}\end{array}$} & \multicolumn{4}{|c|}{ PTV } & \multicolumn{4}{|c|}{ OAR 1} & \multicolumn{4}{|c|}{ OAR2 } \\
\hline & \multicolumn{3}{|c|}{ Average dose } & \multirow[b]{2}{*}{$\gamma$} & \multicolumn{3}{|c|}{ Average dose } & \multirow[b]{2}{*}{$\gamma$} & \multicolumn{3}{|c|}{ Average dose } & \multirow[b]{2}{*}{$\gamma$} \\
\hline & TPS & $\mathrm{COM}$ & $\%$ & & TPS & $\mathrm{COM}$ & $\%$ & & TPS & $\mathrm{COM}$ & $\%$ & \\
\hline 1 & 2805 & 2886 & 2.91 & 0.37 & 797 & 814 & 2.08 & 0.18 & 301 & 308 & 2.25 & 0.09 \\
\hline 2 & 2709 & 2779 & 2.51 & 0.32 & 776 & 741 & 4.51 & 0.26 & 296 & 282 & 4.73 & 0.13 \\
\hline 3 & 2576 & 2648 & 2.79 & 0.57 & 925 & 948 & 2.41 & 0.23 & 421 & 431 & 2.45 & 0.14 \\
\hline 4 & 2706 & 2768 & 2.27 & 0.38 & 937 & 956 & 2.01 & 0.19 & 486 & 497 & 2.33 & 0.13 \\
\hline 5 & 2738 & 2803 & 2.4 & 0.38 & 964 & 982 & 1.91 & 0.2 & 503 & 514 & 2.29 & 0.13 \\
\hline 6 & 2656 & 2713 & 2.15 & 0.33 & 885 & 901 & 1.74 & 0.17 & 463 & 473 & 2.15 & 0.11 \\
\hline 7 & 2664 & 2733 & 2.6 & 0.43 & 954 & 974 & 2.1 & 0.21 & 497 & 509 & 2.4 & 0.13 \\
\hline 8 & 2576 & 2639 & 2.45 & 0.39 & 1018 & 1031 & 1.3 & 0.16 & 459 & 466 & 1.4 & 0.09 \\
\hline 9 & 2755 & 2786 & 1.1 & 0.28 & 1073 & 1061 & 1.1 & 0.16 & 504 & 500 & 0.7 & 0.09 \\
\hline 10 & 2724 & 2781 & 2.07 & 0.33 & 999 & 1019 & 2.03 & 0.19 & 544 & 557 & 2.36 & 0.04 \\
\hline
\end{tabular}

$\lambda$ - average $3 \mathrm{D}$ gamma

were less than 0.6, recommend by Visser et al., (2013).

\section{Discussion}

Many schwannomas can be removed with minimally invasive surgery. However, this is dependent upon the age, overall physical health of the patient, the size and location of the tumor. For the past 10 years, radiation treatment for vestibular schwannoma has been increasingly used as an alternative to microsurgery because it is claimed that it results in high rates of control and elimination of the operative morbidity and because early outcomes were better for patients having stereotactic radiotherapy compared with surgical resection (Kapoor et al., 2011). Tumor control rates reported in linear accelerator series, where the stereotactic irradiation has been given in fractionated mode, are on par with the reported outcome in the largest radio-surgery series using one fraction (Gamma Knife). This study reported the treatment of 10 patients with large cerebral acoustic schwannoma located in eloquent areas. Volumetric modulated arc based hypofractionated stereotactic radiotherapy was administered using conventional linear accelerators. Many studies have shown that VMAT reduced treatment time compared to fixed field IMRT, tomotherapy, cyberknife and gammaknife. Wolff et al., (2010) reported that RapidArc based SRS has shortened treatment time with better conformity. Adding flattening filter free (FFF) beam to VMAT based HSRT further reduces the treatment time. The purpose of this study was to assess the dosimetric benefits of this new technique. The regimen of $25 \mathrm{~Gy}$ in 5 fractions was considered as adequate in the present investigation from a conservative point of view. Kapoor et al., (2010) have shown long term follow up of 496 vestibular schwannoma patients treated with fractionated stereotactic radiotherapy, had good local control and "no further treatment required". Many studies have shown very high local control rates as well as minimal toxicity by delivering $12 \mathrm{~Gy}$ in single fraction for small AS and located in non-eloquent areas. Biological effective dose (BED) of 12Gy in 1 fraction was $60 \mathrm{~Gy}(\alpha / \beta-3 \mathrm{AS})$, where as BED for $25 \mathrm{~Gy}$ in 5 fractions was $67 \mathrm{~Gy}$. From a biological point of view HSRT might have some advantage in comparison to SRS in terms of acute complications and of tumor control rate for lesions larger than 10cc. In SRS, brain necrosis represents the most important late toxicity. $\mathrm{V}_{10 \mathrm{~Gy}}$ and $\mathrm{V}_{12 \mathrm{~Gy}}$ were most important independent predictors of both symptomatic and asymptomatic radionecrosis (Minniti et al., 2011). To avoid brain necrosis, hypofractioned stereotactic treatment were delivered for large brain tumors $(>10 \mathrm{cc})$.

Precise position of patient was vital criteria in stereotactic treatment, delivering high dose per fraction using small fields. Before treatment delivery patient position was verified using CBCT. Ingrosso et al., (2012) have reported that in HSRT, CBCT improves the accuracy of the treatment delivery reducing set-up uncertainty, giving the possibility of 3-dimensional anatomic information in the treatment position. Abaciogle et al., (2014) demonstrate that, compared to a treatment time of 1 hour for gamma knife (SRS), the same delivery can be completed in less than 5 minutes with RapidArc (FFF). The clinical follow-up available for the ten patients presented here was too short to derive indications on long term toxicity or control rates, but it is adequate to outlook early acute complications occurring within the first months after treatment. With a mean follow-up of about 6 months, none of the patients developed acute toxicity.

In stereotactic treatments, there will be very high gradient dose distribution inside the PTV volume. MLC shaped fields have more geometry and dosimetric uncertainties than those of the circular cones. Li et al., (2004) reported that large errors are often caused by a small setup error or measuring point displacement from the central ray of the beam. Before measurements, CBCT was performed to ensure to precise position of detector at isocentre. The measuring dose at the iso-center of small fields, volume averaging will result in an underestimation of the actual dose. Due to this fact, for all 10 patients irrespective of PTV volume CC13 chamber underestimates the calculated iso-center dose. High-spatial resolution detectors were the preferred choice for stereotactic measurements. PSD has $1 \mathrm{~mm}$ spatial resolution showing promising tool for stereotactic treatments. Chamber positioning is very important when using smaller volume because they are more sensitive to geometrical errors within the treatment fields. Gagnon et al., (2012) have reported, the presence of a non-water equivalent dosimeter in a small field such as an ion chamber having a smaller 
Shanmugam Thirumalai Swamy et al

or greater electronic density than water will decrease or increase the lateral charge particle equilibrium artificially and affect the dose readout. Hounsfield variation along the sensitive volume of PSD was significantly lesser than A14 and CC13. Due to above rationale, for all ten patients, PSD shows less deviations compared with other two detectors ( $\mathrm{p}=0.021$ for PSD $v s \mathrm{~A} 14, \mathrm{p}<0.005$ for PSD vs CC13). Leakage current of PSD was more than A14 and $\mathrm{CC} 13$, subsequently leakage current was corrected by subtracting leakage charge (without beam-on). In general, Exradin W1 plastic scintillator detector will be better choice for small filed dosimetry due to its high spatial resolution of $1 \mathrm{~mm}$, nominal sensitive volume of $0.002 \mathrm{~cm}^{3}$ and near water equivalence. However, further in depth study has to be performed to know the effect of leakage, angular dependence and Cerenkov radiation. The purpose of the dose computation in COMPASS is to provide an independent dose calculation engine in order to cross-check the dose calculated by the TPS (Boggula et al., 2010). The study results of average gamma and average dose difference between Eclipse TPS calculated and COMPASS computed were comparable with the earlier reported values by others (Boggula et al. 2010; Visser et al. 2013). Although beam data remains identical for COMPASS and Eclipse, the minor variation in dose distribution was due to difference in beam modeling especially in penumbra and buildup region and in-homogeneity corrections between two different calculation algorithms (AAA and CCC/S) The VMAT QA methodology described here is neither unique nor ubiquitous, and the ability to deliver a safe VMAT does not simply require VMAT QA tests to pass a given tolerance; however, the selection of a tolerance level should be meaningful by assessing gradient index in chamber volume, target shape and MLC segments.

In conclusion, volumetric modulated arc based hypofractionated stereotactic radiotherapy produces clinically acceptable plans for large sized acoustic schwannoma located in eloquent areas. Adding flattening filter free beam reduces treatment time, which improves patient comfort. Pretreatment treatment quality assurance was mandatory when delivering high dose using small beamlets. Point dosimetry and secondary TPS verification provides confidence of treatment delivery and dose calculation accuracy. In point dose measurement, high spatial resolution and near water equivalence characteristics of plastic scintillator detector provides superior results compared to ion chambers.

\section{References}

Abacioglu V, Ozen Z, Yilmaz Y, et al (2014). Critical appraisal of RapidArc radiosurgery with flattening filter free photon beams for benign brain lesions in comparison to GammaKnife, a treatment planning study. Radiation Oncology, 9, 119.

Benedict SH, Cai J, Libby B, et al (2010). SRT and SBRT, Current practices for QA dosimetry and 3D, Journal of Physics, Conference Series, 250, 012057.

Benedict SH, Yenice KM, Followill D, et al (2010). Stereotactic body radiation therapy, The report of AAPM task group 101. Med Phy, 37, 4078-101.
Boggula R, Lorenz F, Mueller L, et al (2010). Experimental validation of a commercial $3 \mathrm{D}$ dose verification system for intensity-modulated arc therapies. PhysMed Biol, 55, 5619-33.

Ekambaram V, Vealyudham R, Swaminathan S, et al (2015). Planning aspects of volumetric modulated arc therapy and intensity modulated radio therapy in carcinoma left breast - A comparative study. Asian Pac J Cancer Prev, 16, 1633-36

Gagnon JC, Riault DT, Guillot M, et al (2012). Dosimetric performance and array assessment of plastic scintillation detectors for stereotactic radiosurgery quality assurance. Med Phys, 39, 429-36

Ingrosso G, Micheli R, Fedele D, et al (2012). Cone-beam computed tomography in hypofractionated stereotactic radiotherapy for brain metastases. Radiation Oncology, 7,54.

Kappor S, Batra S, Carson K, et al (2011). Long-term outcomes of vestibular schwannomas treated with fractionated stereotactic radiotherapy, An institutional experience. Int $J$ Radiat Oncol Biol Phys, 81, 647-53.

Li S, Rashid A, He S, Djajaputra A (2004). A new approach in dose measurement and error analysis for narrow photon beams beamlets shaped by different multileaf collimators using a small detector. Med Phys, 31, 2020-32.

Minniti G, Clarke E, Lanzetta, et al (2011). Stereotactic radiosurgery for brain metastases, analysis of outcome and risk of brain radionecrosis. Radiation Oncology, 6, 48.

Subramanian S, Srinivas C, Ramalingam K, et al (2012). Volumetric modulated arc-based hypofractionated stereotactic radiotherapy for the treatment of selected intracranial arteriovenous malformations, Dosimetric report and early clinical experience. Int J Radiat Oncol Bio Phys, 82, 1278-84.

Swamy ST, Radha CA, Kathirvel M, et al (2014). Feasibility study of deep inspiration breath-hold based volumetric modulated arc therapy for locally advanced left sided breast cancer patients. Asian Pac J Cancer Prev, 15, 9033-38.

Thirumalai SS, Anuradha C, Kathirvel M, et al (2014). Pretreatment quality assurance of volumetric modulated arc therapy on patient CT scan using indirect 3D dosimetry system. Int J Cancer Ther Onco, 2, 20416.

Visser R, Wauben DJ, de Groot M, et al (2013). Efficient and reliable 3D dose quality assurance for IMRT by combining independent dose calculations with measurements. Med Phys, 40, 021710.

Wang H, Chang RJ, Xiao F (2012). Hypofractionated stereotactic radiotherapy for large arteriovenous malformations. Surg Neurol Int, 3, 105-10.

Wolff HA, Wagner DM, Christiansen H, et al (2010). Single fraction radiosurgery using Rapid Arc for treatment of intracranial targets. Radiation Oncology, 5, 77.

Xin Y, Wang JY, Li L, et al (2012). Dosimetric verification for primary focal hypermetabolism of nasopharyngeal carcinoma patients treated with dynamic intensity-modulated radiation therapy. Asian Pac J Cancer Prev, 13, 985-9. 